\title{
STRUCTRUAL INTEGRITY OF A WIND TUNNEL BALANCE
}

\author{
R. Karkehabadi and R. D. Rhew \\ NASA LaRC, Hampton, VA
}

\section{INTRODUCTION}

The National Aeronautics and Space Administration (NASA) Langley Research Center (LaRC) has been designing strain-gage balances for utilization in wind tunnels since its inception. The utilization of balances span over a wide variety of aerodynamic tests. A force balance is an inherently critically stressed component due to the requirements of measurement sensitivity. Research and analyses are done in order to investigate the structural integrity of the balances as well as developing an understanding of their performance in order to enhance their capability.

Maximum loading occurs when all 6 components of the loads are applied simultaneously with their maximum value allowed (limit load). This circumstance normally does not occur in the wind tunnel. However, if it occurs, is the balance capable of handling the loads with an acceptable factor of safety?

LaRC Balance 1621 was modeled and meshed in PATRAN for analysis in NASTRAN. For a complete analysis, it is necessary to consider all the load cases as well as use dense mesh near all the edges. Because of computer limitations, it is not possible to have one model with the dense mesh near all edges. In the present study, a dense mesh is limited to the surface corners where the cage and axial sections meet. Four different load combinations are used for the current analysis. Linear analysis is performed for each load case. In the case where the stress value is above linear elastic region, it is necessary to perform nonlinear analysis.

It is also important to investigate the variables limiting the structural integrity of the balances. In order to investigate the possibility of modifying the existing balances to enhance the structural integrity, some modifications are done on this balance. The structural integrity of the balance after modification is investigated.

\section{APPLIED LOAD}

The limit loads for this balance are shown in Table 1. The loads given are in the coordinate system shown in Figure 1. Transformation is necessary since the loads are applied at Point $p$ and the values given below are valid for loads applied at the Moment Center.

\begin{tabular}{|c|c|}
\hline $\begin{array}{c}\text { Force and Moment } \\
\text { Components }\end{array}$ & $\begin{array}{c}\text { Force (lb) and Moment } \\
\text { (in-lb) Values }\end{array}$ \\
\hline Axial (Fx) & 500 \\
\hline Side (Fy) & 1800 \\
\hline Normal (Fz) & 3000 \\
\hline Roll (Mx) & 7500 \\
\hline Pitch (My) & 10000 \\
\hline Yaw (Mz) & 4500 \\
\hline
\end{tabular}

Table 1. Maximum Forces and Moments

Four different load combinations are considered and shown below.

Case 1:

$\vec{F}_{x}=-500 \hat{i}$

$\vec{F}_{y}=1800 \hat{j}$

$\vec{F}_{z}=-3000 \hat{k}$

$\vec{M}_{o}=7500 \hat{i}+10000 \hat{j}+4500 \hat{k}$

Case 2:

$\vec{F}_{x}=-500 \hat{i}$

$\vec{F}_{y}=1800 \hat{j}$

$\vec{F}_{z}=3000 \hat{k}$

$\vec{M}_{o}=7500 \hat{i}-10000 \hat{j}+4500 \hat{k}$

Case 3:

$\vec{F}_{x}=-500 \hat{i}$

$\vec{F}_{y}=1800 \hat{j}$

$\vec{F}_{z}=-3000 \mathrm{k}$

$\vec{M}_{o}=-7500 \hat{i}+10000 \hat{j}+4500 \hat{k}$

Case 4:

$\vec{F}_{x}=-500 \hat{i}$

$\vec{F}_{y}=1800 \hat{j}$ 


$$
\begin{aligned}
& \vec{F}_{z}=3000 \hat{k} \\
& \vec{M}_{o}=-7500 \hat{i}-10000 \hat{j}+4500 \hat{k}
\end{aligned}
$$

The loads from the Moment Center (MC) are transformed to Point $\mathrm{P}$, as shown below for each case:

$$
\begin{aligned}
& \vec{M}_{p}=7500 \hat{i}+22300 \hat{j}+11880 \hat{k} \text { Case } 1 \\
& \vec{M}_{p}=7500 \hat{i}-22300 \hat{j}+11880 \hat{k} \text { Case } 2 \\
& \vec{M}_{p}=-7500 \hat{i}+22300 \hat{j}+11880 \hat{k} \text { Case } 3 \\
& \vec{M}_{p}=-7500 \hat{i}-22300 \hat{j}+11880 \hat{k} \text { Case } 4
\end{aligned}
$$

\section{LINEAR ANALYSIS}

\section{A. GLOBAL MODEL}

The present model was generated and meshed using PATRAN. The section of the material where the load is applied is not modeled in order to reduce the number of elements. One of the load cases that produce regions of high stress on the balance, Load case 1, is used for the analysis of the present section. This load case has all components positive except axial force (Fx) and normal force $(\mathrm{Fz})$. The components of the load applied at Point $\mathrm{p}$ are shown in the load section. The results are expected to be accurate away from the applied load due to St. Venant's principal.

Because of computer limitation, the model was meshed using a little less than 200,000 elements, all 10-node tetrahedral elements, tet10. The result of this run is used for Global-Local analysis. Global-Local analysis is used in order to obtain more accurate results for the selected parts of the model. With this method, a desired section (local model) is meshed and loads and boundary conditions are obtained from the results of the global model analysis. A 3-D view of the meshed model used in the present work is shown in Figure 2.

The Von-Mises stress and principal stresses for the axial section near the applied load are shown in Figures 3 and 4. As the figures indicate, maximum Von-Mises stress (267 KSI) and principal stress (274 KSI) both occur near the applied load. It should be mentioned that the values shown are for the coarse mesh and to find more accurate results, more elements are needed, particularly near the corners.

\section{B. LOCAL ANALYSIS OF AXIAL SECTION NEAR THE APPLIED LOAD}

There are many corners on the balance and in order to capture the stress gradient, a dense mesh is required near these corners until convergence occurs. In order to use more elements, because of the computer limitations, the global model has to be divided into smaller local sections.

A section near the applied load was extracted and meshed, as shown in Figure 5. The results of the global model were used as the boundary conditions for this local model. The VonMises stress is shown in Figure 6. Since the result is above the linear region, a nonlinear analysis is required.

\section{MODEL FOR NONLINEAR ANALYSIS}

The results from the linear analysis indicate that the stress value is above the linear elastic region; hence, a nonlinear analysis is required. Because NASTRAN tet10 elements do not allow some nonlinearities in the version used here, the model used for a nonlinear analysis is meshed with 4 node tetrahedral, tet 4 . The balance is meshed with almost 400,000 tet 4 elements. A dense mesh is used near the end of the axial sections, Figure 7. A linear analysis was performed on the balance with the new mesh and all four load cases were considered. If the linear analysis indicates a maximum stress value below yield stress, nonlinear analysis was not performed.

\section{CASE 1 LOADING}

\section{LINEAR ANALYSIS}

The full model is used for the linear and nonlinear analysis. The Von-Mises stress from the linear analysis for the balance is shown in Figure 8. The full model is used and the results from the linear and nonlinear analyses are shown. The maximum stress occurs near the junction of the axial and cage section is shown in Figure 8.

\section{NONLINEAR ANALYSIS}

The Von-Mises stress from the nonlinear analysis for the balance is shown in Figure 9. As expected, the value of the maximum Von-Mises stress dropped in comparison with the linear case.and is below the yield point. 
In order to have an overall view of the stresses everywhere on the balance, Figure 10 is plotted. Figure 10 shows the position versus VonMises stress for all points on the balance. The figure clearly indicates that there are high stress regions and the maximum stress values are localized, two peak stresses occur at the end of the axial sections.

It should be noted that the maximum stress value near other corners might increase as more dense mesh is used near those corners. However, in the present work, the interest is at the end of the axial section.

\section{CASE 2 LOADING}

\section{LINEAR ANALYSIS}

The Von-Mises stress and principal stress from the linear analysis for the balance are shown in Figures 11 and 12. As the figures indicate, the high stress occurs at the end of the axial section.

\section{NONLINEAR ANALYSIS}

The Von-Mises and principal stresses from the nonlinear analysis are shown in Figures 13 and 14. As expected, the maximum stress value for the Von-Mises and principal stresses dropped in comparison with the linear analysis.

In order to have an overall view of the stresses everywhere on the balance for this load case, Figure 15 is plotted. As shown, the high stress values are localized.

\section{CASE 3 AND CASE 4 LOADING}

The results of the analysis for the load cases 3 and 4 are shown. Because the value of stress for both cases is below yield, a nonlinear analysis was not performed. These two load cases are examples of the cases that produce stresses below yield.

\section{LINEAR ANALYSIS}

Figures 16 and 17 are the Von-Mises and principal stresses from the linear analysis for Load Case 3. Figures 18 and 19 are the results from Load Case 4. The Von-Mises stress from the linear analysis for the balance is shown in Figure 18, and the principal stress is shown in Figure 19. As the figures indicate, the stresses are below yield.

\section{MODIFICATION OF SOME HIGH STRESS REGIONS}

Balance 1621 was modeled in PATRAN and meshed with tet 10 . One load combination is used in the present analysis. The model is meshed with 287,426 elements. The result of this analysis was used as a boundary condition on a local model. In order to obtain local models for analysis, sections from the balance were extracted and meshed. The local model is a portion of the balance near the applied load, Figure 20. This model is meshed with 251,983 tet10 elements. Figure 21 shows the maximum Von-Mises stress for this section.

By increasing the fillet size, it is possible to reduce the high-localized stresses. There was a cut of .05 inch on the surface and a fillet radius of .05 inch was added. This model was meshed with 273,050 tet10 elements. The result of the global model was used for the local model. Figure 22 is a section of the balance near the applied load. There are 238,644 elements on this model. The results obtained from this model are shown in Figure 23. As the figure indicates, the maximum Von-Mises stress lowered after modification. It should be noted that the maximum value shown in Figure 23 is not for the same location as shown in Figure 21, it is near the T-section.

It is important to have a dense mesh, especially near the corners. NASTRAN was used for the analysis on a PC computer. The present runs reached the vicinity of the maximum computer resources available.

\section{CONCLUSION}

Not all the balances and not all the load combinations need nonlinear analysis. For the existing balances, Pro/Mechanica, NASTRAN, or other Finite Element software can be used for the linear analysis. The analysis needs to be done for all the load cases. If the maximum stress value obtained is beyond yield, either a change in the design needed or the maximum loads allowed need to be reduced. The reduction of the force can be in certain components. This is practical since in most cases the wind tunnel tests do not require maximum value of the loads on all of the components. In some cases the wind tunnel test requires some components pass the maximum value while the maximum value of the other components are not needed and can be reduced. 
For the balances that will be designed in the future, increasing the size of the fillets help to relieve some of the local high stresses. GlobalLocal analysis was used for the balance 1621 before and after modification. The density of the mesh used the maximum capability of the computer available for this work. The modification resulted into a lower stress value in the region that was investigated. The results are encouraging. It is an indication that performance of existing balances may be enhanced by some modifications.

\section{Z}

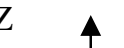

Figure 1. Balance 1621 with its coordinate axis located at the Moment Center

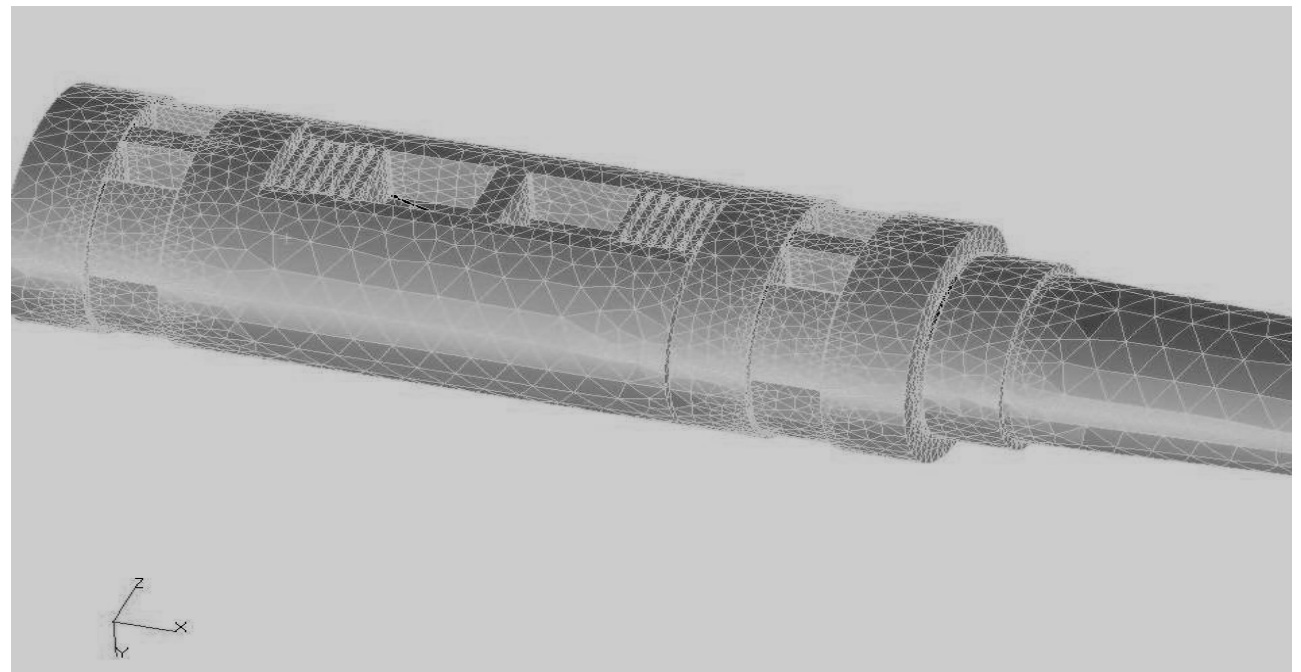

Figure 2. A 3-D view of the balance modeled and meshed in PATRAN 


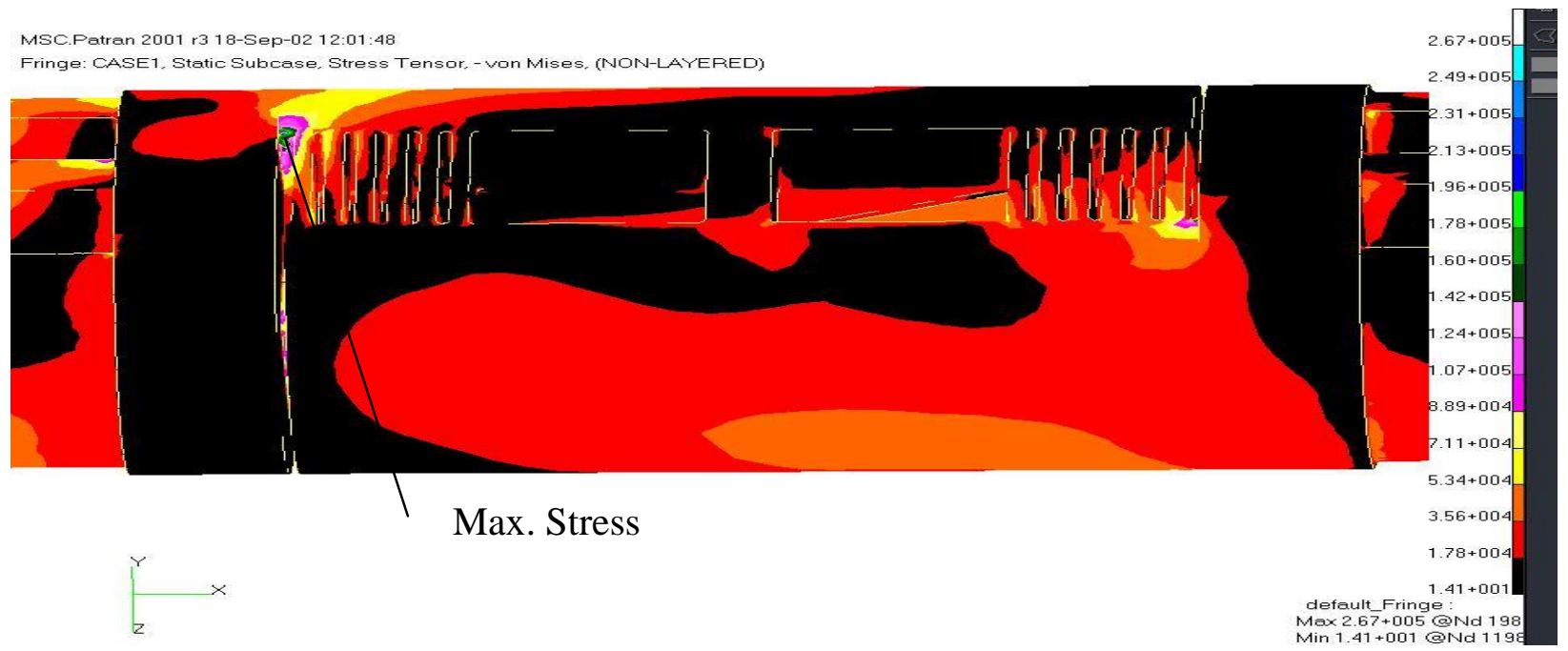

Figure 3. The Von-Mises stress for the balance, load case 1

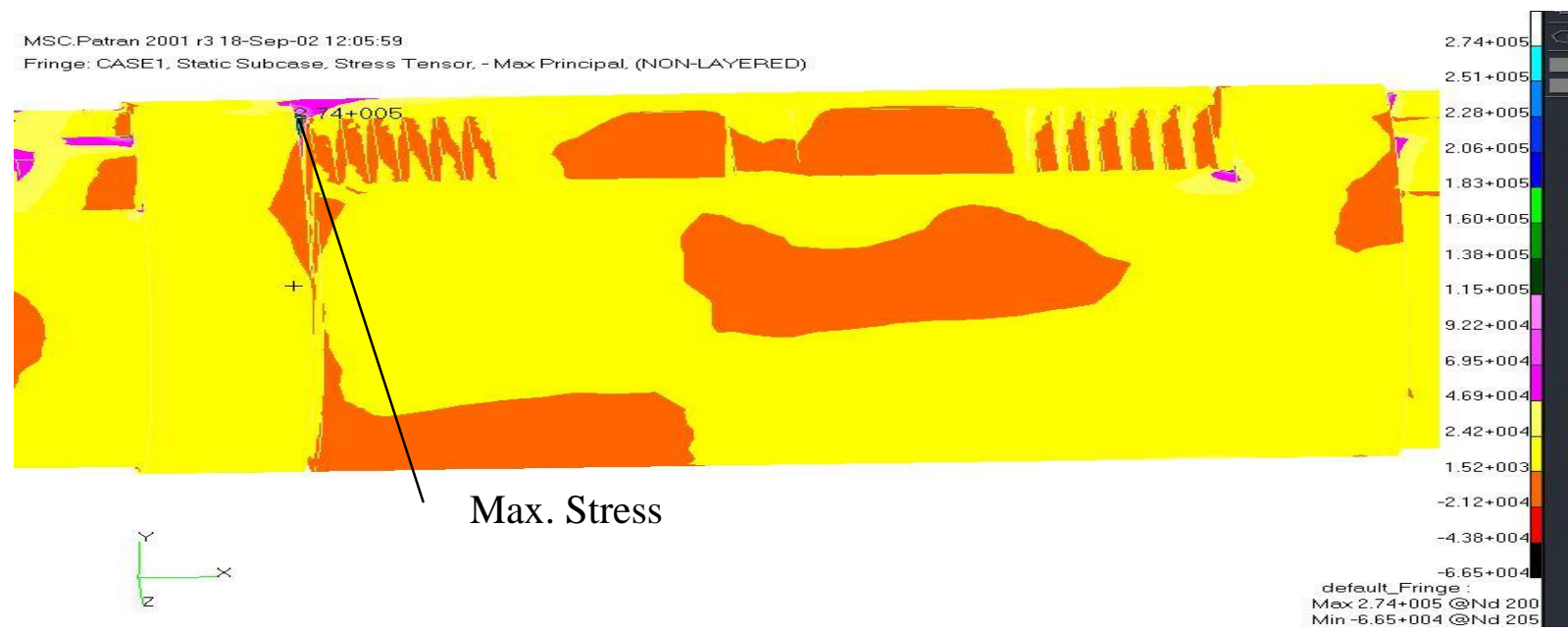

Figure 4. The principal stress for the balance, load case 1

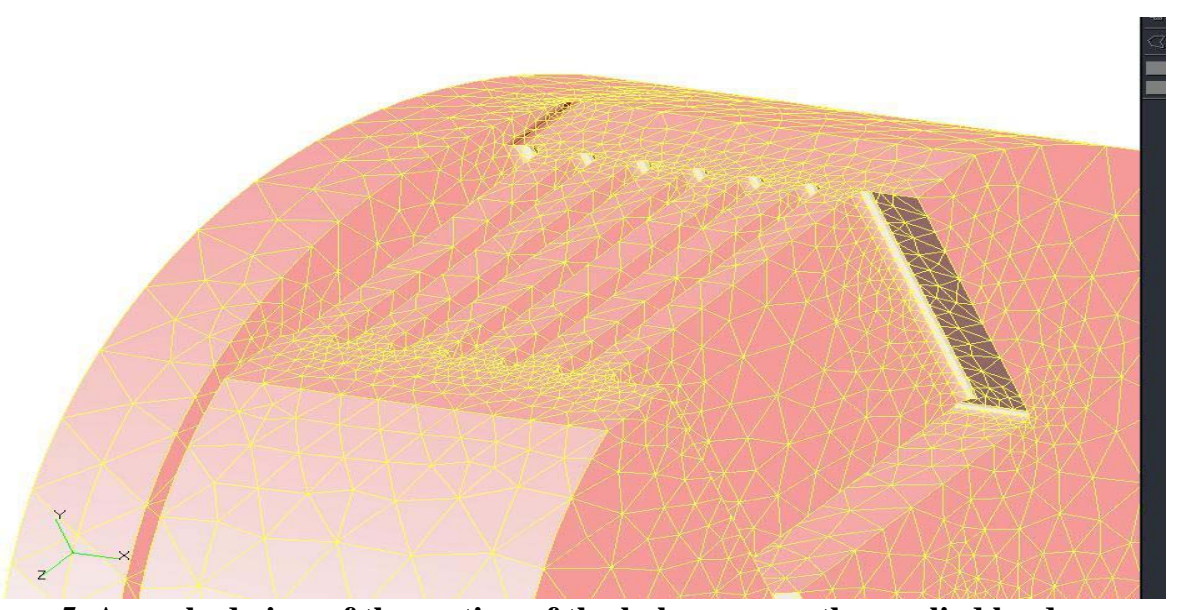

Figure 5. A meshed view of the section of the balance near the applied load 


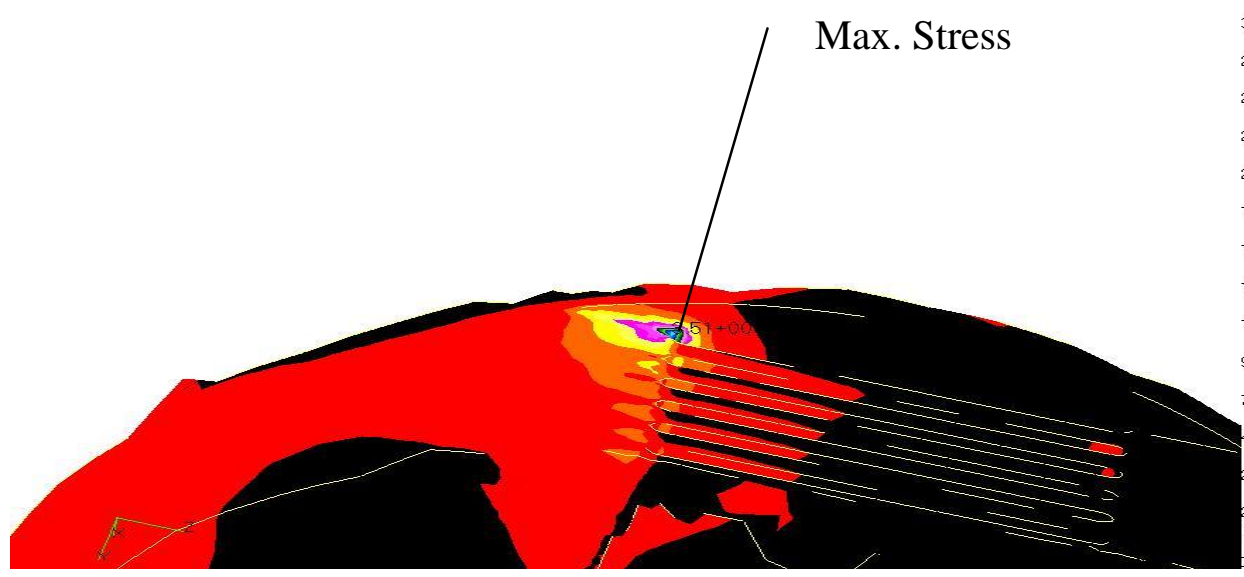

Figure 6. Von-Mises stress for the section of the balance near the applied load

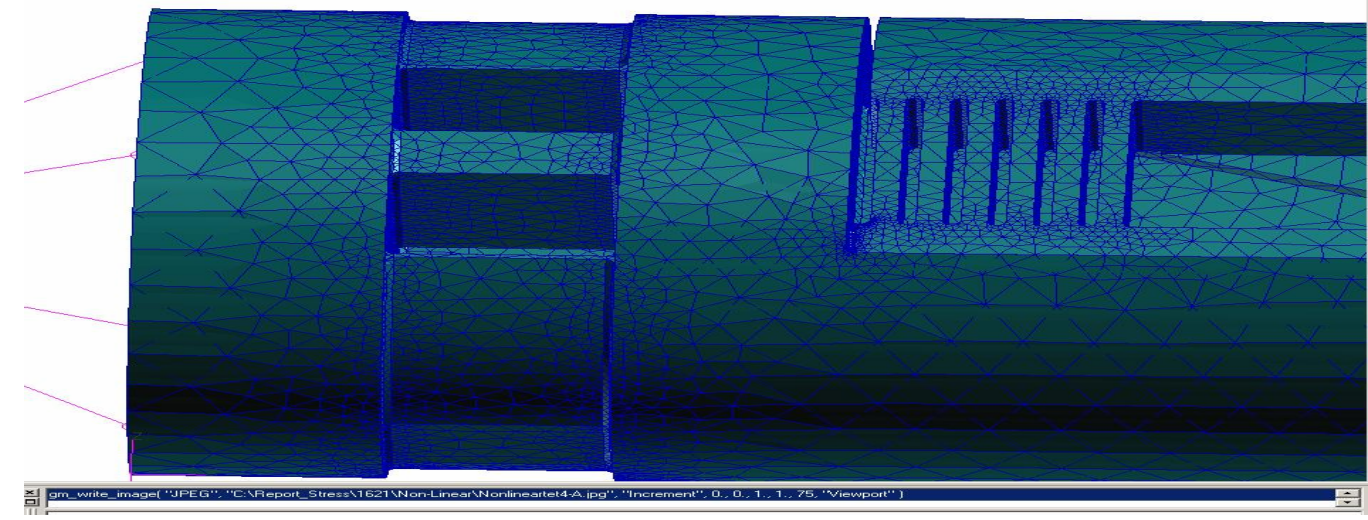

Figure 7. A close-up view of the end of the axial section

MSC.Patran 2001 r3 17-Oct-02 13:24:09

Fringe: Case1. Static Subcase. Stress Tensor, - von Mises, (NON-LAYERED)

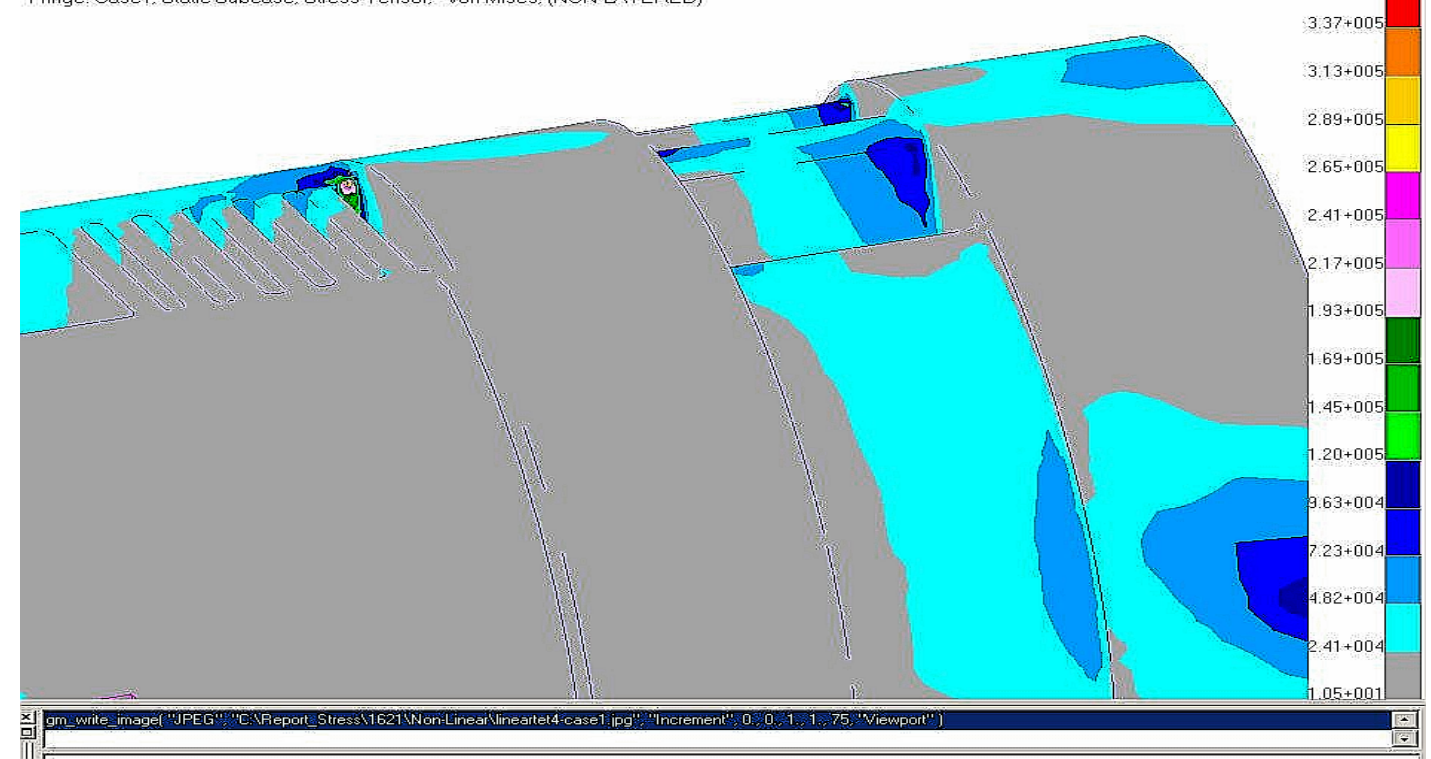

Figure 8. The Von-Mises stress for the balance from the linear analysis, Load Case 1 


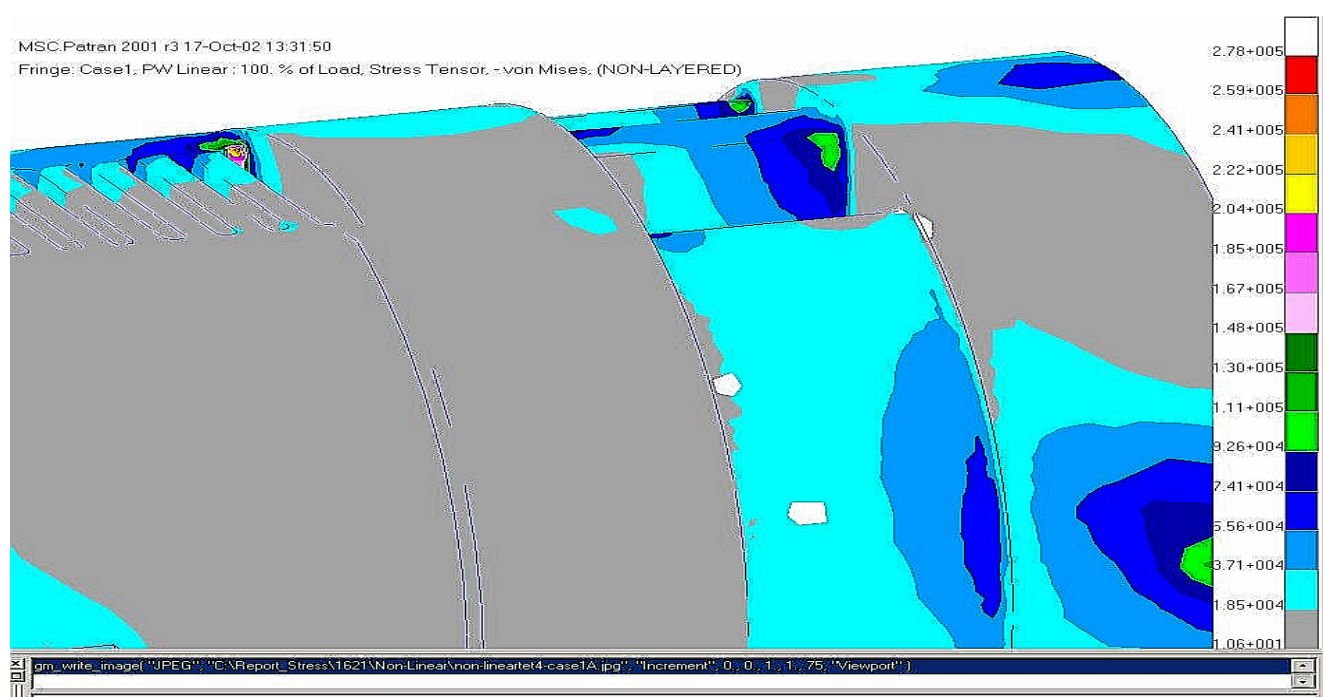

Figure 9. The Von-Mises stress for the balance from the nonlinear analysis, Load Case 1

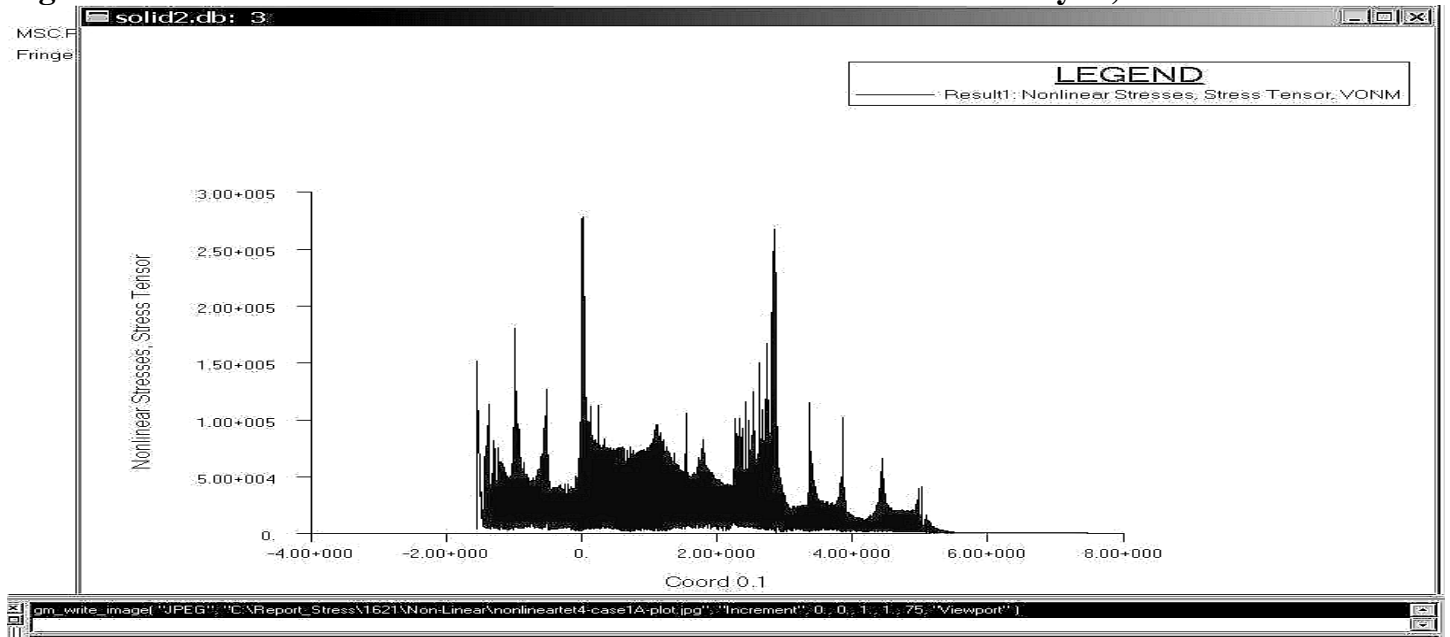

Figure 10. Axial position vs. Von-Mises stress for all points on the balance, Load Case 1

MSC.Patran 2001 r3 17-OCt-02 14:07:10

Fringe: Case2. Static Subcase_2: Stress Tensor,-(NON-LAYERED) NONM)

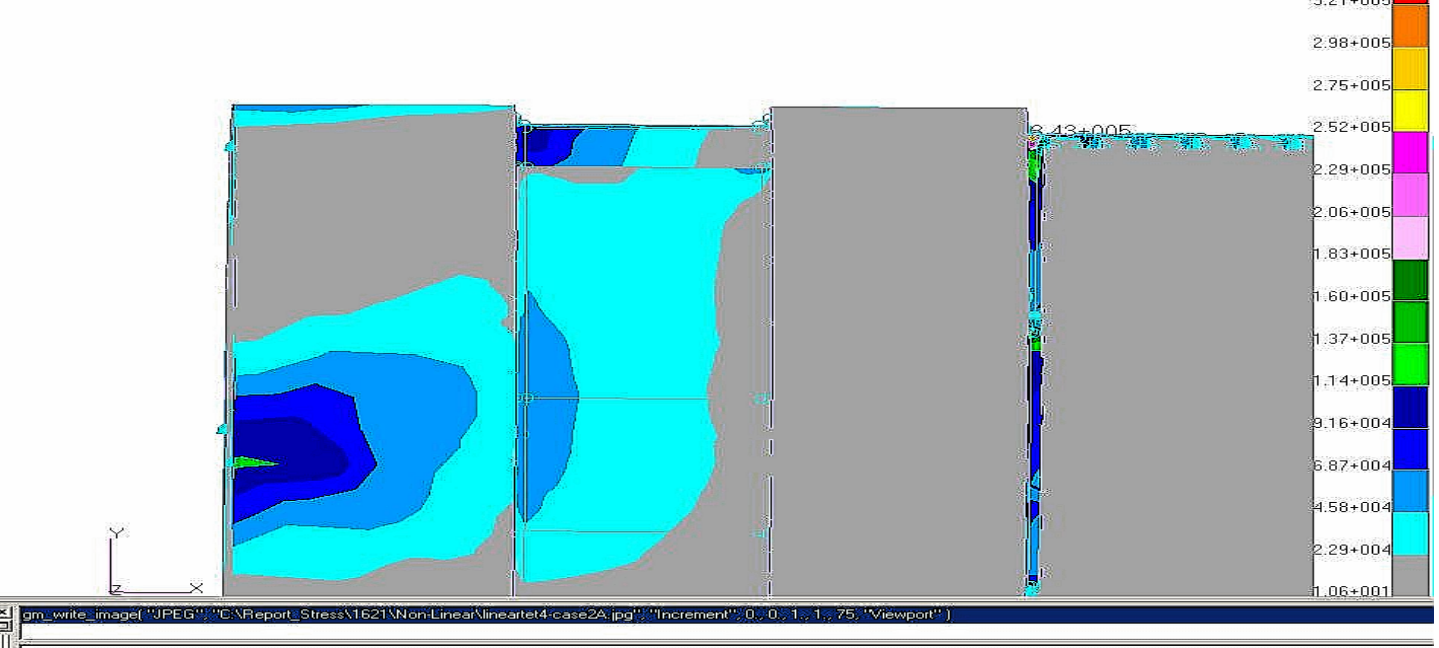

Figure 11. The Von-Mises stress for the balance from the linear analysis, Load Case 2 


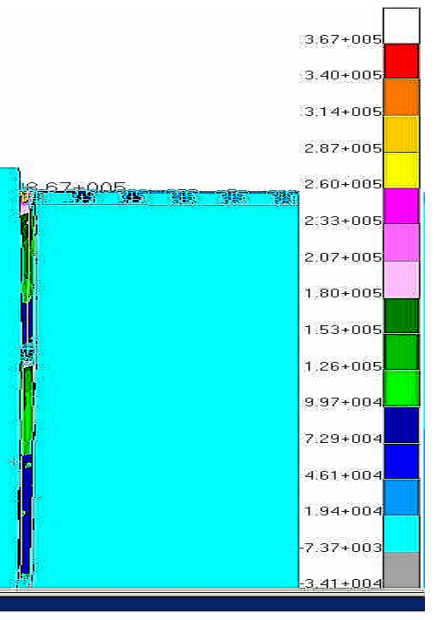

\section{Figure 12. The principal stress for the balance from the linear analysis, Load Case 2}

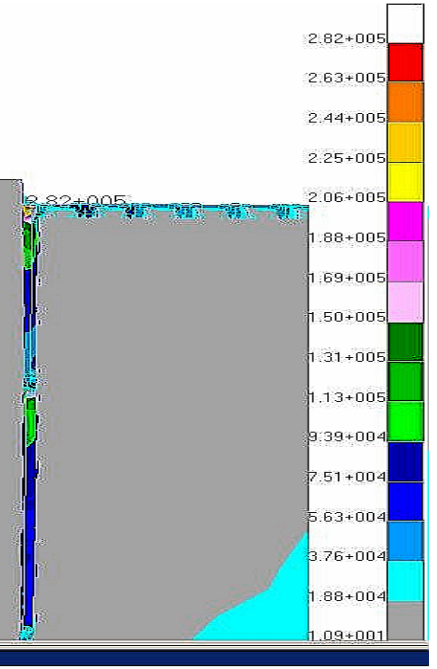

Figure 13. The Von-Mises stress for the balance from the nonlinear analysis, Load Case 2

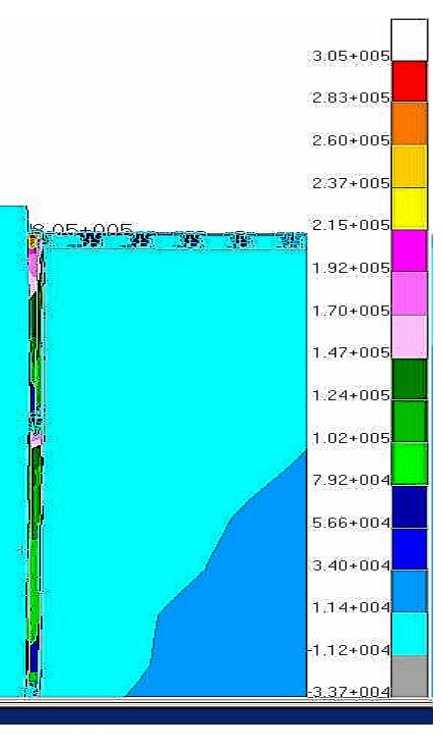

Figure 14. The principal stress for the balance from the nonlinear analysis, Load Case 2 


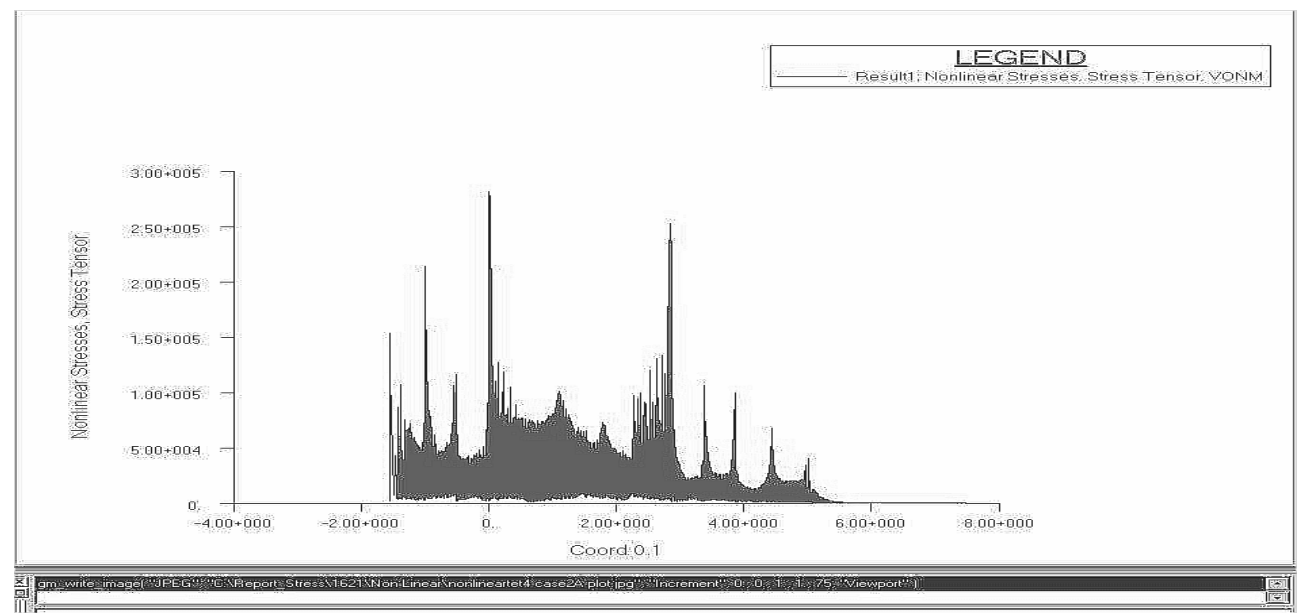

Figure 15. Position vs. Von-Mises stress for all points on the balance, Load Case 2

MSC.Patran 2001 r3 17-Oct-02 14:22:05

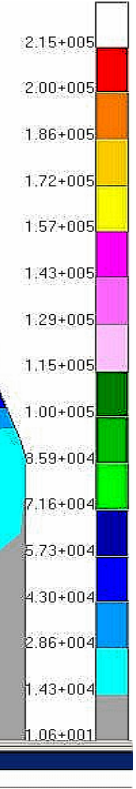

Figure 16. The Von-Mises stress for the balance from the linear analysis, Load Case 3

MSC.Patran 2001 r3 17-Oct-02 14:19:43

Fringe: Case3, Static Subcase_2: Stress Tensor, -(NON-LAYERED) (MAJOR)

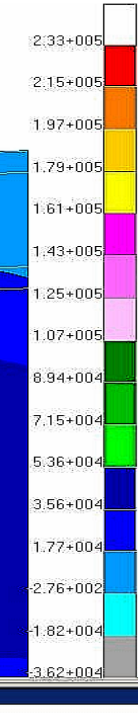

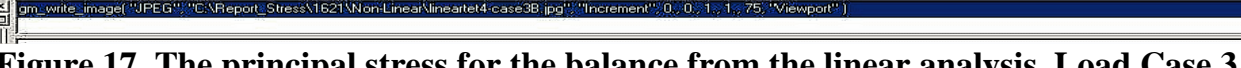

Figure 17. The principal stress for the balance from the linear analysis, Load Case 3 


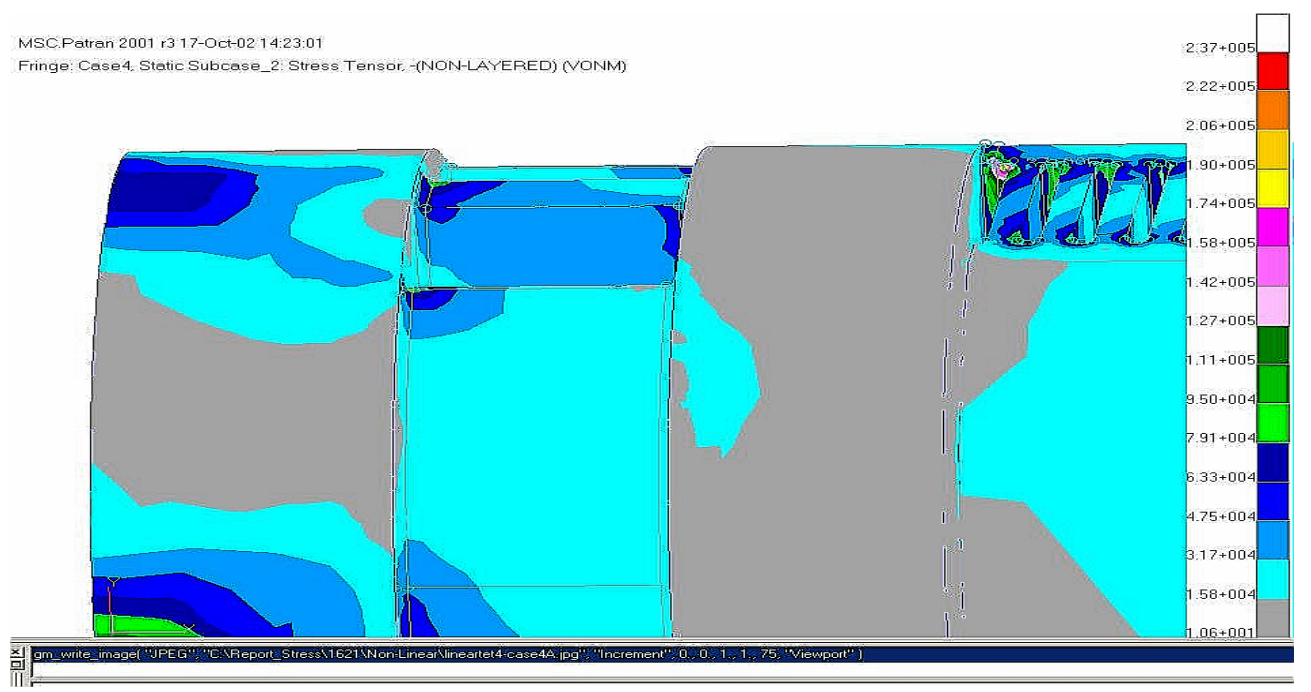

Figure 18. The Von-Mises stress for the balance from the linear analysis, Load Case 4

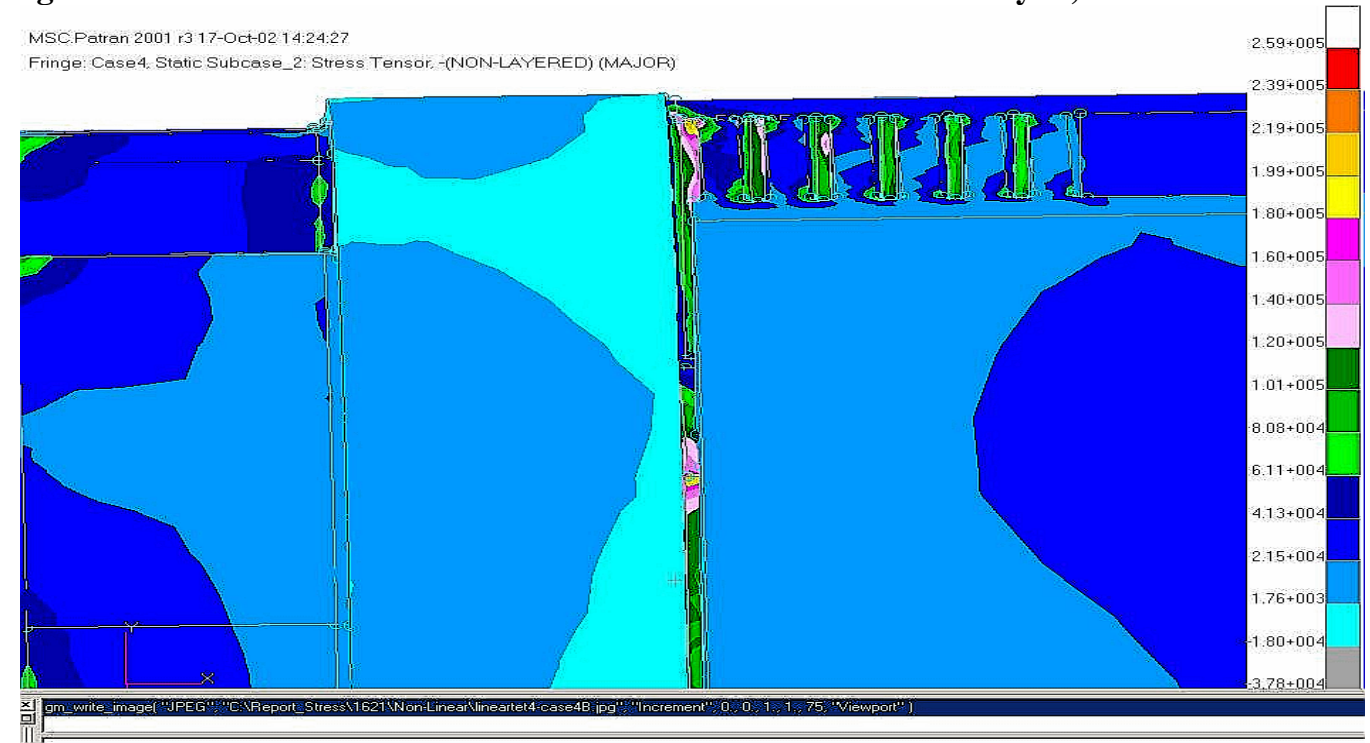

Figure 19. The principal stress for the balance from the linear analysis, Load Case 4

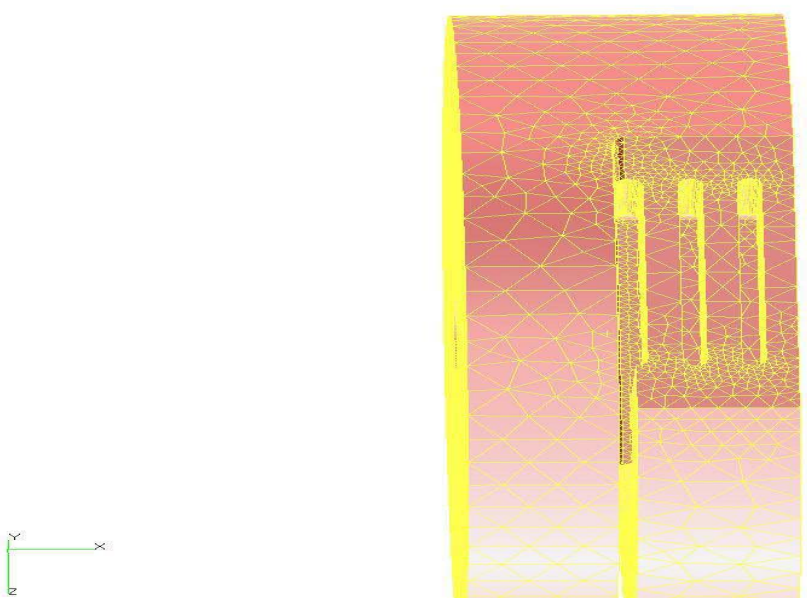

Figure 20. Meshed local PATRAN model before modification 


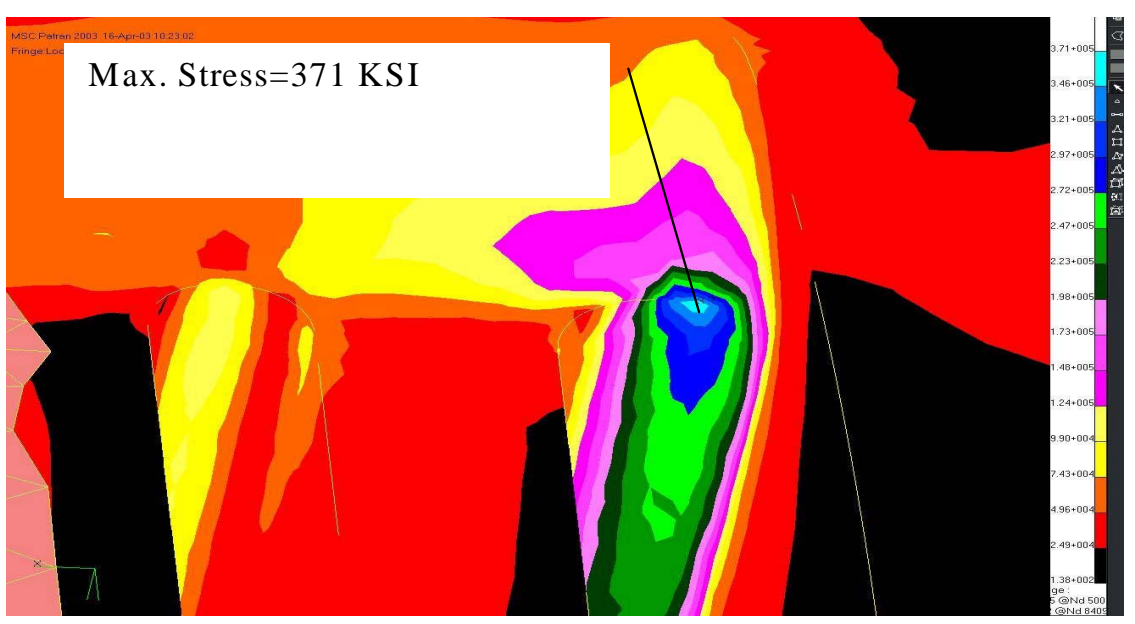

Figure 21. Von-Mises stress for local model before modification

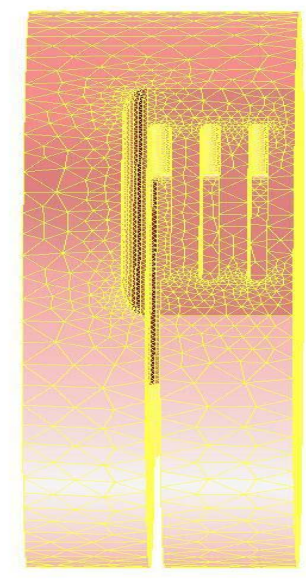

Figure 22. Meshed local model after modification

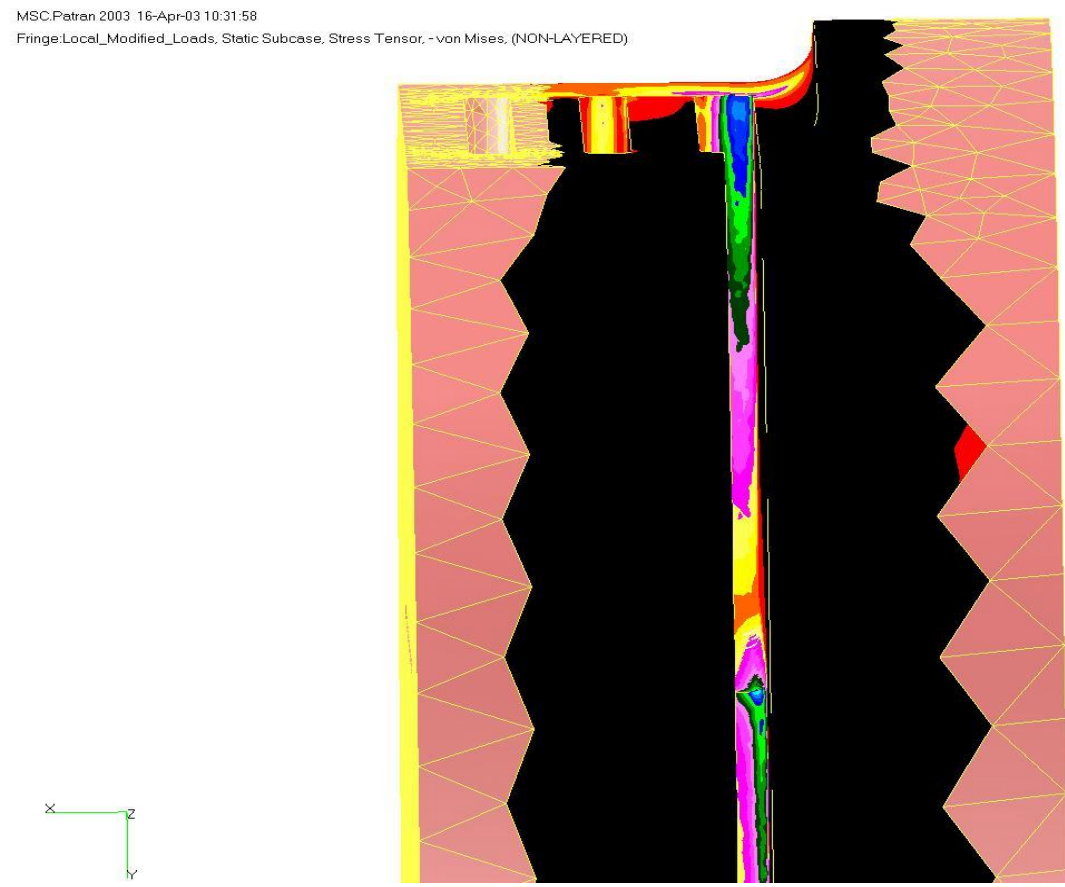

Figure 23. Von-Mises stress for local model after modification

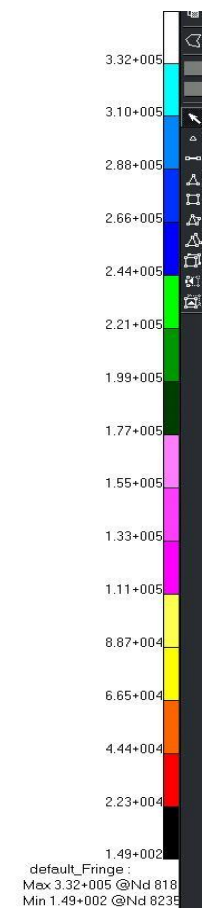

